\title{
Research on diagnosis method of automobile fault based on fuzzy $\mathrm{H}$ net Xulong Xiang \& Lian Pan \\ College of information science and engineering, School of Wuhan University of Science and Technology
}

Keywords: fuzzy $\mathrm{H}$ net; diagnosis of automobile fault; data table.

\begin{abstract}
This paper is studied according to the method of auto fault diagnosis and proposes a data table structure of fuzzy diagnosis model based on fuzzy $\mathrm{H}$ net with the presenting of the reasoning process. Aimed at the uncertainty and fault fuzzy appearing in automobile, fuzzy $\mathrm{H}$ net is used to the fault diagnosis of auto, which effectively reduces the complexity of fuzzy reasoning. Finally, through the experiment of vehicle failure to test the possibility of the model.
\end{abstract}

\section{Introduction}

In the process of long-term use, due to various environmental factors and failure, the auto parts or the whole function of the car decline or lose.Car trouble tend to be complex, the process of fault propagationis continuous and concurrency.In order to be able to quickly determine the cause of the problem and the location and high quality, with the minimum consumption of labor, objective evaluation of the car and the status of the technology, also with the reason for the advanced automobile fault diagnosis technology, the study of fault diagnosis theory is very urgent.Fuzzy $\mathrm{H}$ net is the further development of petri nets, its knowledge representation and information processing ability was stronger than the fuzzy petri net. There is only one due to the fuzzy $\mathrm{H}$ net node, both the information storage and information processing functions, so the network analysis, modeling, storage can be greatly simplified. To this end, this paper proposes a fuzzy reasoning algorithm of the data table structure of FHN.The algorithm firstly defines a FHN five-metal-group, and then gives a fuzzy production rule type, based on the data table structure of FHN reasoning process, summarizes the fuzzy reasoning algorithm of FHN, finally through the car trouble example of this method is verified.

\section{The fuzzy $H$ network analysis}

Fuzzy $\mathrm{H}$ net network is a kind of fuzzy Hstructure, and it has only a node, namely fuzzy $\mathrm{H}$ nodes.The functions of fuzzy $\mathrm{H}$ nodes both position and have the function of transfer nodes, as shown in figure 2.1.

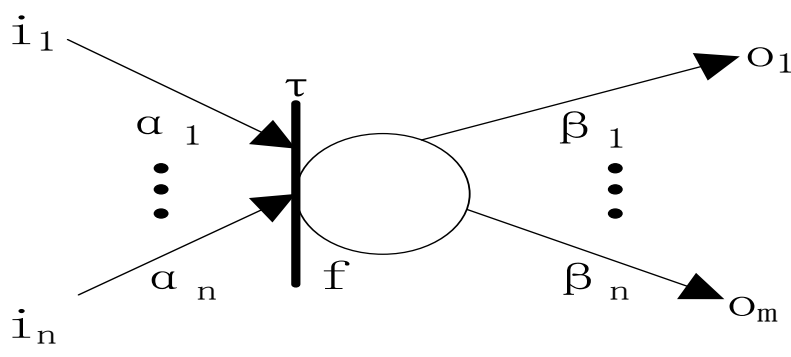

Fig 2.1 Fuzzy H net node

In figure 2.1, $n \geq 0 m \geq 00 \leq \alpha, \beta \leq 1$ shows to the edge of the connection strength $i=1,2, \cdots, n$; $j=1,2, \cdots, m 0 \leq \tau \leq \infty$ callednode activation threshold, which is defined in the input of a transfer function. Fuzzy $\mathrm{H}$ net is a fuzzy graph composed of the node to connect to the edge, as shown in figure 2.2. By a vertical bar into the end of the connecting line is called the input line, and cable arise out of the circle at one end is called the output line. No input connection of nodes is called the fuzzy $\mathrm{H}$ net input, no output connection of nodes is called the fuzzy $\mathrm{H}$ net output. 


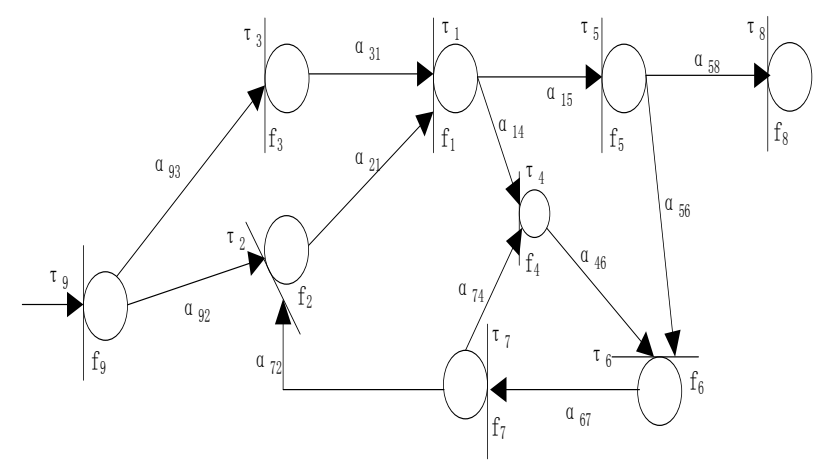

Fig 2.2 Fuzzy H net structure chart

Definition 1.1 used for knowledge representation of fuzzy $\mathrm{H}$ net can be defined as a five-metal-group:

$$
H=\{P, R, F(p), \tau(p), \quad \mathrm{S}(p)\}(1)
$$

The $P$ a finite set of fuzzy $\mathrm{H}$ node $R$ is a fuzzy relation of $P \times P$ fuzzy representation in $\mathrm{H}$ between nodeshave to set connection edges. $F(p)$ Is an image in $P$, it will be in the fuzzy $\mathrm{H}$ node mapping for an increasing non negative real function, called the transfer function of $P$ node. $\tau(p), S(p)$ are real functions defined on $P$ and value for the $[0, \infty)$.

\section{The fuzzy H net PRODUCTION RULES}

The solution of the fuzzy $\mathrm{H}$ net first to construct fuzzy production rule, the fuzzy production rules are used to describe the uncertainty of the relations between events, is a kind of the IF - THEN the structure of fuzzy conditional sentences, commonly used production rules have the following three categories:

(1) As shown in figure 2.1, fuzzy production rules are described as: If A and B, then C, and the ignition conditions as follows:

$\min \{$ A. $\mu \times \alpha$, B. $\mu \times \alpha\} \geq C . \tau$

(2) The figure 2.1 the fuzzy production rule can be described as: If $\mathrm{A}$ or $\mathrm{B}$, then $\mathrm{C}, C F=u$ and the ignition conditions as follows:

$\max \{A . \mu \times \alpha, B . \mu \times \alpha\} \geq C . \tau$

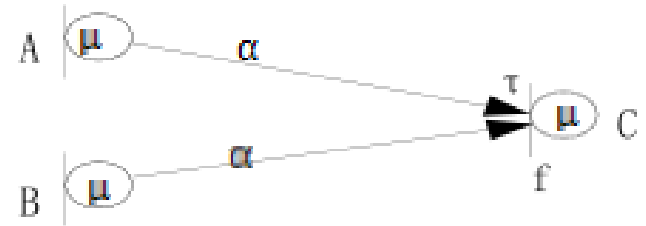

Fig 2.1 Fuzzy H net rule graph (1)

(3) As shown in figure 2.2, fuzzy production rules are described as: If $\mathrm{A}$, then $\mathrm{B}$ and $\mathrm{C} C F=u$ ignition conditions as follows: $A . \mu \times \alpha \geq \max \{B . B \tau, C . C \tau\}$

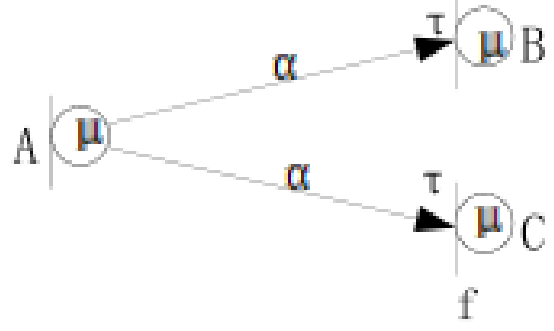

Fig2.2 Fuzzy H net rule graph (2)

In FHN, each node is corresponded to a rule, the fuzzy $\mathrm{H}$ nodes of the input is the precondition of rule conditions, the output is the result of the rules, the mapping function to the credibility of the corresponding rules, the rules of the match and FHN corresponding fuzzy $\mathrm{H}$ the ignition of a node. It's said you just need two kinds of graph pattern. Therefore, this paper defined the fuzzy $\mathrm{H}$ net 
production rules into two types: (1) If $\mathrm{A}$ and $\mathrm{B}$, then $\mathrm{C}$; (2) If $\mathrm{A}$ or $\mathrm{B}$, then $\mathrm{C} C F=u$ (2) If $\mathrm{A}$ or $\mathrm{B}$, then $\mathrm{C} C F=u$ it reduces the complexity of the subsequent reasoning process.

\section{FHN reasoning algorithm}

\subsection{FHN data table structure}

FHN fuzzy reasoning algorithmproposed in this paper is through the establishment of data table structure, by using the data table to reasoning, and literature is given based on numerical and symbolic fusion reasoning algorithm compared to more intuitive and convenient.The data of this table contains the three types of information: (1) the fuzzy $\mathrm{H}$ accessibility node assemble $R S\left(p_{i}\right)$; (2) the mapping function of the fuzzy $\mathrm{H}$ node;(3) fuzzy $\mathrm{H}$ node immediately accessibility node assemble $\operatorname{IRS}\left(p_{i}\right)$.

Table 1 FHN date table

\begin{tabular}{|c|c|c|c|}
\hline Type & $R S\left(p_{i}\right)$ & $\operatorname{IRS}\left(p_{i}\right)$ & $F\left(p_{i}\right)$ \\
\hline Type1 & $p_{1}, p_{2}, p_{3}, \ldots, p_{n}$ & $p_{1}, p_{2}, p_{3}, \ldots, p_{n}$ & $\mu_{1}$ \\
\hline \multirow[t]{3}{*}{ Type2 } & $p_{1}$ & $p_{1}$ & $\mu_{2}$ \\
\hline & $\ldots$ & $\ldots$ & $\ldots$ \\
\hline & $p_{n-1}$ & $p_{n-1}$ & $\mu_{n}$ \\
\hline
\end{tabular}

Mappingthe two kinds of fuzzy production rule to the data in table 1, can providethe convenience for the next step of reasoning.

\subsection{The reasoning mechanism}

Before the operation, Fuzzy $\mathrm{H}$ net needs for initialization processing, that according to the acquired information and facts to give each node a positive number greater than or equal to 0 , the initial value is called the operation of the fuzzy $\mathrm{H}$ node id number. In the running process, the calculation of each node of the input node of the input intensity, the intensity of the input is the identification number of input nodes and the corresponding input connection strength of a dual function. When the target Class rules match 1 rule

$Y\left(\mu_{k}, \alpha_{i}\right)=\min \{A . \mu \times \alpha, B . \mu \times \alpha\} ;$

When the target Class rules match 2 rule

$Y\left(\mu_{k}, \alpha_{i}\right)=\max \{A . \mu \times \alpha, B . \mu \times \alpha\}$.

The complete computation of input intensity of each node, then calculated the value of the transfer function of the corresponding nod $f$ is $(\mathrm{P})$, when

$f \geq \tau$;

(Threshold), the node will enter the ignition, and then the strength of each input node ID number is subtracted from the corresponding input line $Y\left(c_{k}, \alpha_{i}\right)$, and at the same time will be the node with ID number plus with $f$. By this kind of operation with one step by another, till the end of the operation.

\section{FAULT DIAGNOSIS EXAMPLE}

The stop of the automobile engine has many reasons, such as the starting system, security system, electric control system, electronic control fuel injection system and fire engine failure, etc. This paper is aimed at the fuel oil supply caused by abnormal start-up failure analysis with the following rules:

If the carburetor fault $\left(P_{1}\right)$ action, then pump oilabnormal $\left(P_{5}\right)$ action $u=0.8$;

If the fuel tank switch is not open $\left(P_{2}\right)$ action, then the oil pump oil supply abnormality $\left({ }_{P_{5}}\right)$ action $u=0.9$;

If the tubing plug $\left(P_{3}\right)$ action, then oil field not free not to $\left(P_{6}\right)$ action $u=1.0$; 
If insufficient storage oil tank $\left(P_{4}\right)$ action, then oil field not free not to $\left(P_{6}\right)$ action $u=0.8$;

The If $\left(P_{5}\right)$ node, then don't start the engine $\left(P_{7}\right)$ action $u=0.8$;

If $\left(P_{6}\right)$ node, then engine fault, don't start $\left(P_{6}\right) u=0.8$. 2:

The corresponding fuzzy $\mathrm{H}$ net model diagram as shown in figure 4.1, FPN data are shown in table

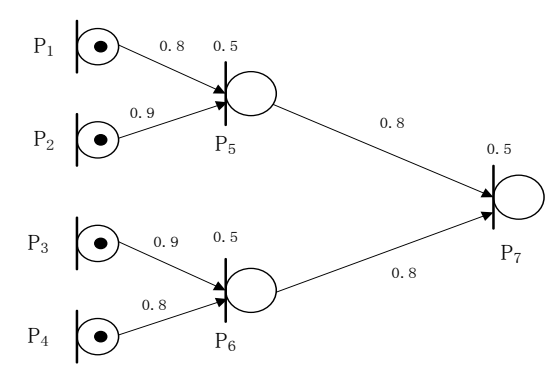

Fig4.1 Fuzzy H net instance model

Table 2 Fuzzy H net instance date

\begin{tabular}{c|c|c|c|c|c}
\hline$R S\left(p_{i}\right)$ & $\left(P_{l}\right)$ & $\left(P_{2}\right)$ & $\left(P_{3}\right)$ & $\left(P_{4}\right)$ & $\left(P_{5}, P_{6}\right)$ \\
\hline $\operatorname{IRS}\left(p_{i}\right)$ & $\left(P_{5}\right)$ & $\left(P_{5}\right)$ & $\left(P_{6}\right)$ & $\left(P_{6}\right)$ & $\left(P_{7}\right)$ \\
\hline$F\left(p_{i}\right)$ & 0.8 & 0.9 & 0.9 & 0.8 & 0.8 \\
\hline
\end{tabular}

System initialization and give the corresponding node assignment, according to the figure 4.1 shows that the initial state identified as $S=\left[\begin{array}{lllllll}1 & 1 & 1 & 1 & 0 & 0 & 0\end{array}\right]^{T}$; System model is up and running, if the category 1 production rules, the running results for $P_{7}=0.64$;If use category 2 production rule, as the operation result $P_{7}=0.72$. System to run over, to get results $S=\left[\begin{array}{lllllll}0 & 0 & 0 & 0 & 0 & 0 & 2\end{array}\right]^{T}$, shows the abnormal cause fuel system can't start the engine.

\section{Conclusion}

Car trouble is expressed by the fuzzy $\mathrm{H}$ net of causality, logic relations between system knowledge is more simple, and clearer, by given the FHN data table, the reasoning process is more simple and intuitive, which is easy to implement; on the basis of reasoning mechanism, the data table givensolves the concurrency and fuzziness in the process of automobile fault diagnosis problem. Through the fault of fuel system to supply abnormal instance, show that the method is an effective method of auto fault diagnosis test.

\section{References}

[1] He Xingui. Fuzzy H net [J]. Journal of computer, 1995, 18 (2): 153-157.

[2] Kong Fansen, Dong Yinping. Fault tree knowledge of automobile fault diagnosis and reasoning based on [J]. Automotive engineering, 2001, 23 (3): 209-213

[3] Koch I, Junker B H, Heiner M. Application of Petri net theory for modeling and validation of the sucrose breakdown pathway in the potato tuber [J]. Bioinformatics, 2005, 21(7): 1219-1226

[4] Qiu Jing, Jia Hongliang. Fault diagnosis expert system of processing equipment based on fuzzy $\mathrm{H}$ net model and object orientedmethod fusion, China mechanical engineering 10.11(1999): 1246-1249.

[5] Zhang Baiyi, Cui Shangsen. Fault diagnosis method of automobile of fuzzy Petri net based on [J]. Journal of Chang'an University (NATURAL SCIENCE EDITION), 2008, 28 (2): 93-96. 